OPEN ACCESS

Edited by: Aditi Banerjee,

University of Maryland, Baltimore, United States

Reviewed by:

Cem Parlak,

Başkent University, Turkey

Onita Bhattasali, Kaiser Permanente Los Angeles Medical Center, United States

${ }^{*}$ Correspondence:

Chang-Chun Hsiao cchsiao@mail.cgu.edu.tw

Eng-Yen Huang

hey1200@cgmh.org.tw

Specialty section: This article was submitted to

Radiation Oncology,

a section of the journal

Frontiers in Oncology

Received: 13 December 2021 Accepted: 24 January 2022 Published: 23 February 2022

Citation:

Huang $C-C$, Chuang I-C, Su Y-L, Luo H-L, Chang Y-C,

Chen J-Y, Hsiao C-C and Huang E-Y (2022) Prognostic Significance of Galectin-1 but

Not Galectin-3 in Patients

With Lung Adenocarcinoma

After Radiation Therapy.

Front. Oncol. 12:834749. doi: 10.3389/fonc.2022.834749

\section{Prognostic Significance of Galectin-1 but Not Galectin-3 in Patients With Lung Adenocarcinoma After Radiation Therapy}

\author{
Chun-Chieh Huang ${ }^{1,2}$, I-Chieh Chuang ${ }^{3}$, Yu-Li Su ${ }^{4}$, Hao-Lun Luo ${ }^{5}$, Ya-Chun Chang ${ }^{6,7}$, \\ Jo-Ying Chen ${ }^{1}$, Chang-Chun Hsiao ${ }^{2,7^{*}}$ and Eng-Yen Huang ${ }^{1,8^{*}}$ \\ ${ }^{1}$ Department of Radiation Oncology, Kaohsiung Chang Gung Memorial Hospital and Chang Gung University College of \\ Medicine, Kaohsiung, Taiwan, ${ }^{2}$ Graduate Institute of Clinical Medical Sciences, College of Medicine, Chang Gung University, \\ Taoyuan, Taiwan, ${ }^{3}$ Department of Anatomical Pathology, Kaohsiung Chang Gung Memorial Hospital and Chang Gung \\ University College of Medicine, Kaohsiung, Taiwan, ${ }^{4}$ Division of Hematology-Oncology, Department of Internal Medicine, \\ Kaohsiung Chang Gung Memorial Hospital and Chang Gung University College of Medicine, Kaohsiung, Taiwan \\ ${ }^{5}$ Department of Urology, Kaohsiung Chang Gung Memorial Hospital and Chang Gung University College of Medicine, \\ Kaohsiung, Taiwan, ${ }^{6}$ Department of Internal Medicine, Kaohsiung Municipal Min-Sheng Hospital, Kaohsiung, Taiwan, \\ 7 Division of Pulmonary and Critical Care Medicine, Kaohsiung Chang Gung Memorial Hospital and Chang Gung University \\ College of Medicine, Kaohsiung, Taiwan, ${ }^{8}$ School of Traditional Chinese Medicine, Chang Gung University College of \\ Medicine, Taoyuan, Taiwan
}

Introduction: To investigate the role of tumor galectin-1 and galectin-3 in patients with lung adenocarcinoma after definitive radiation therapy.

Methods: A total of 41 patients with localized lung adenocarcinoma undergoing thoracic radiation therapy without concurrent chemotherapy were enrolled. Their paraffinembedded lung tissues were sent for immunohistochemical staining for galectin-1 and galectin-3. The clinical treatment outcomes, including overall (OS), locoregional progression-free (LRPFS), and distant metastasis-free (DMFS) survivals, were evaluated. Univariable and multivariable Cox regression analyses were applied.

Results: Overexpression of tumor galectin-1 and galectin-3 were found in $26.8 \%$ and $19.5 \%$ of patients, respectively. Overexpression of tumor galectin-1 was the most significant prognosticator to predict worse LRPFS in both univariable $(p=0.007)$ and multivariable analyses $(p=0.022)$. Besides, patients with overexpression of tumor galectin-1 had a trend of worse OS $(p=0.066)$ than those with low expression in multivariable analysis, and worse DMFS $(p=0.035)$ in univariable analysis. The overexpression of tumor galectin-3 had no significant effect on survival outcomes.

Conclusions: The overexpression of tumor galectin-1, but not galectin-3, is associated with poor LRPFS of patients with lung adenocarcinoma after thoracic radiation therapy. Future research on the mechanism of galectin-1 affecting radiation response in lung adenocarcinoma may be worth exploring.

Keywords: galectin-1, galectin-3, lung adenocarcinoma, radiation therapy, radiation response biomarkers 


\section{INTRODUCTION}

Lung cancer is one of the leading cancers with high incidence and mortality rates in the world. In Taiwan, lung adenocarcinoma is the most common histological type of lung cancer. According to the Taiwan Cancer Registry Annual Report 2018 (1), lung adenocarcinoma accounted for $58 \%$ in men and $88 \%$ in women with a new diagnosis of lung cancer. The clinical managements to treat lung adenocarcinoma to get better tumor control and survival outcomes are of great value.

Radiation therapy is an important local treatment modality for lung adenocarcinoma $(2,3)$. Short course of stereotactic ablative radiation therapy is a standard local therapy for medically inoperable early-stage lung adenocarcinoma (4). Long course of definitive radiation therapy plays a major role in the locoregional treatment of locally advanced lung adenocarcinoma (5). For metastatic lung adenocarcinoma, local radiation therapy is recommended for palliation of symptoms and can be used for consolidation in oligometastatic or oligo-residual tumors (6). For brain metastasis, both radiosurgery and whole brain irradiation are important treatment options to control intracranial tumors (7). Therefore, the method to improve radiation response and the study on the radioresistance in lung adenocarcinoma are necessary.

Galectins are a family of proteins with binding specificity for beta-galactoside sugars by glycosylation and fifteen mammalian galectins have been identified till now. Galectin-1 is a homodimeric protein encoded by the LGALS1 gene, and galectin-3 is the only chimeric lectin of the galectin family encoded by the LGALS3 gene. Both galectin-1 (8-10) and galectin-3 (11-13) have been found to be involved in tumor development and progression, such as cell proliferation, invasion, and metastasis in lung cancer. As for radiation response, our previous studies first found that galectin-1 was associated with radioresistance in the cervical cancer cell line (14) and was a poor prognostic factor in the patients with squamous cell carcinoma of uterine cervix (15) after definitive radiation therapy. We also proposed that overexpression of galectin-1 was an independent prognosticator in the patients with glioblastoma multiforme (16) after adjuvant radiation therapy, and in the patients with locally advanced esophageal cancer (17) after preoperative chemoradiotherapy. Consequently, in this study, we investigated the prognostic impact of galectin-1 overexpression as well as galectin-3 overexpression on the patients with lung adenocarcinoma after definitive radiation therapy.

\section{MATERIALS AND METHODS}

\section{Patients}

This study was approved by the Chang Gung Medical Foundation Institutional Review Board (IRB) (No. 201800074B0). The need for individual informed consent from each patient was waived by the IRB as minimal risk to the patients who were already diagnosed and treated. This study was initiated in February 2018. A total of 466 patients diagnosed with lung cancer undergoing thoracic radiation therapy at least $50 \mathrm{~Gy}$ in our institution without concurrent chemotherapy between 1987 and 2002 were recorded in our radiation therapy registry database. Patients were staged by thoracic computed tomography including lower neck and upper abdomen area, bone scintigraphy, and brain magnetic resonance imaging.

The following patients were sequentially excluded: 138 with previous thoracic operation, 49 with cytology only or without registry of histopathology type, 226 with non-adenocarcinoma, and 12 with distant metastasis before radiation therapy or inadequate paraffin-embedded tissues for immunohistochemical study. As a result, the pathological tissues of lung adenocarcinoma from 41 patients were enrolled for galectin-1 and galectin-3 immunostainings in this study. Other clinical data including gender, date of birth, initial diagnosis date, first visit date in our department, cancer stage, radiation therapy, neoadjuvant chemotherapy, treatment response, and follow-up status were also acquired for analysis.

\section{Radiation Therapy}

Patients were treated by conventional (27 patients) or threedimensional conformal (14 patients) radiation therapy. The gross tumor volume comprised primary lung tumor and enlarged regional mediastinal lymph nodes with a short diameter of $1 \mathrm{~cm}$ at least. The clinical target volume was gross tumor volume plus 0.5 $\mathrm{cm}$ margin to cover microscopic extension. The margin around clinical tumor volume to develop planning target volume was $0.5-$ $1.0 \mathrm{~cm}$ to cover setup uncertainty and respiratory motion according to lung tumor location. Two to three radiation portals with Cerrobend blocks or multileaf collimators were applied to cover the planning target volume. Most patients were prescribed with a radiation dose of 60-66 Gy in 1.8-2.0 Gy per fraction. After the completion of radiation therapy, the following are carried out: follow-up of chest $\mathrm{x}$-ray and computed tomography within 1-3 months to evaluate treatment response, and then chest $\mathrm{x}$-ray every 1-3 months, computed tomography every 3-6 months, bone scintigraphy and brain magnetic resonance imaging annually, or when clinically indicated.

\section{Immunohistochemistry}

The detailed process of tissue microarray construction and immunohistochemistry has been described as in our previous studies $(15,16)$. Briefly, areas presenting histopathologic characteristics of lung adenocarcinoma were selected on the H\&E slides. The representative areas were marked on the corresponding paraffin block. The tissue cylinders were taken from the selected areas of the donor paraffin block. Then, they were punched into the recipient paraffin block by tissue-arraying instruments. Several $1-\mu \mathrm{m}$-thick sections were cut and mounted on the slides. They were stained with antibodies for galectin-1 (HPA000646, rabbit to human; Sigma-Aldrich, St. Louis, MO) and for galectin-3 (Santa Cruz B2C10).

The immunostaining features of galectin- 1 and galectin- 3 in the tumor cells and surrounding stroma were evaluated by a single pathologist who were blinded to the clinical treatment outcomes. The percentages of positive staining for galectin- 1 and galectin-3 in the tumor part and stroma part were scored as follows: 0 for $<10 \%$ positive staining; 1 for $10 \%$ to $<40 \%$ positive 
staining; 2 for $40 \%$ to $<70 \%$ positive staining; 3 for at least $70 \%$ positive staining (Figures 1 and 2). Both score 2 and score 3 were designated as overexpression of galectin- 1 or galectin- 3 except that score 1 of galectin-3 in stroma was also regarded as overexpression due to lack of score 2 or score 3 of galectin-3 expression in stroma.

\section{Statistical Analysis}

The clinical treatment outcomes, including overall (OS), locoregional progression-free (LRPFS), and distant metastasisfree (DMFS) survivals were calculated using the Kaplan-Meier method. OS was calculated from the date of initial diagnosis, and both LRPFS and DMFS were calculated from the date of starting radiation therapy. Univariable and multivariable Cox regression analyses were used to check possible prognostic factors of OS, LRPFS, and DMFS. The factors, including gender, age, $\mathrm{T}$ stage, $\mathrm{N}$ stage, tumor galectin-1, stroma galectin-1, tumor galectin-3, stroma galectin-3, radiation dose, and neoadjuvant chemotherapy, were evaluated in both univariable and multivariable analyses. The results for prognostic factors were presented as hazard ratio (HR) with $95 \%$ confidence interval
(CI). The $p$-values were two-sided and less than 0.05 was considered to be statistically significant. The statistical analyses were calculated by SPSS Statistics 22.0 (IBM Corp., Armonk, NY).

\section{RESULTS}

The characteristics of patients are listed in Table 1. Most patients were diagnosed as locally advanced stage (T3-T4 stage: 78\% and N2-N3 stage: $68.3 \%$ ). Overexpression of tumor galectin-1 and galectin-3 were found in $26.8 \%$ and $19.5 \%$ of patients, respectively. Overexpression of stroma galectin-1 and galectin-3 was found in $85.4 \%$ and $39.0 \%$ of patients, respectively.

The median follow-up time was 10.2 months (range, 3.0204.7 months; mean, 20.0 months). Nearly all patients were followed up until death, except for one patient due to loss of follow-up after 7.1 months. There were 3 patients with first recurrence at locoregional area and 14 patients with first recurrence at distant site. The median OS, LRPFS, and DMFS were 10.3 months, 9.0 months, and 6.4 months, respectively. The
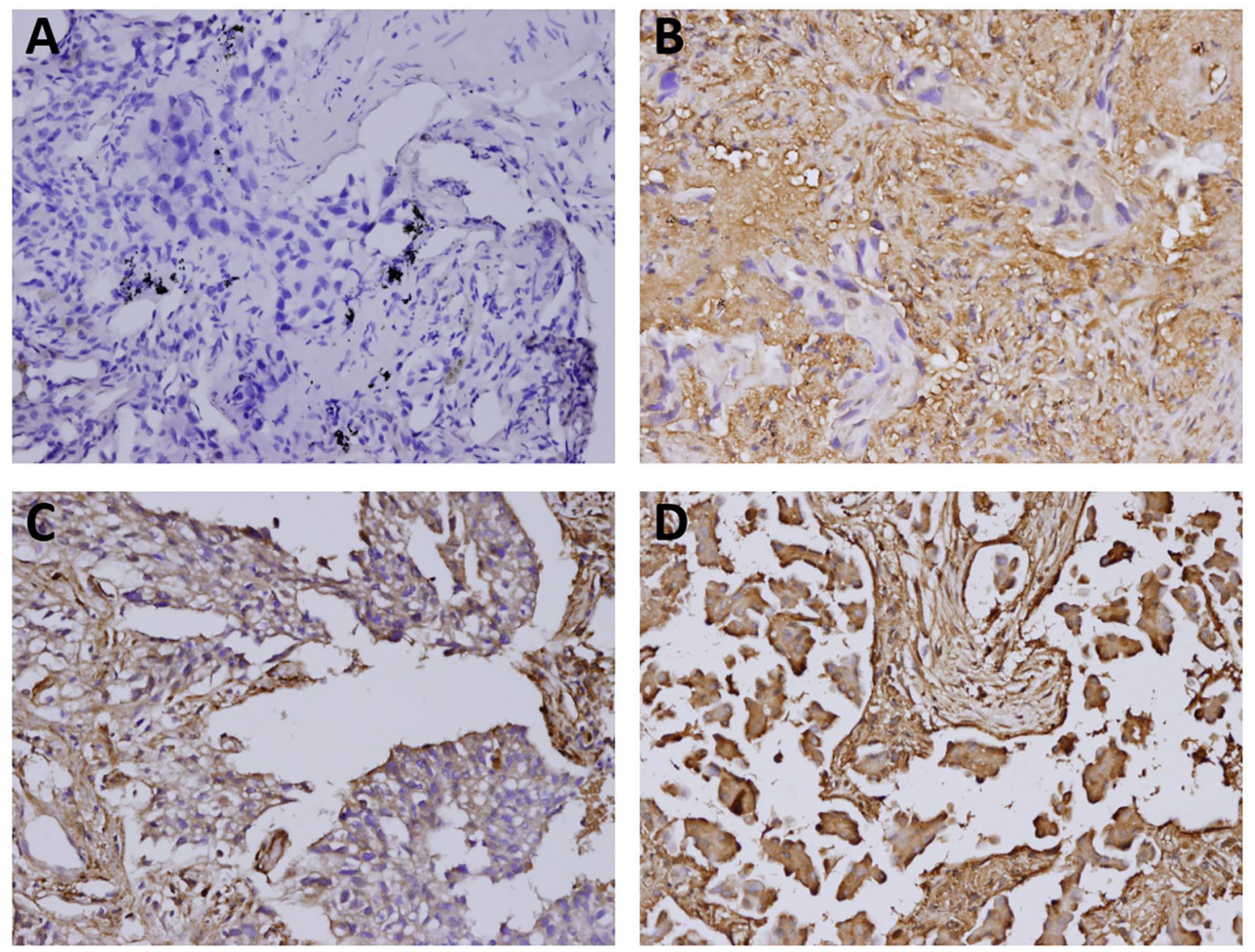

FIGURE 1 | The immunostaining features of tumor galectin-1 expression. The percentages of positive staining for galectin-1 in the tumor part were scored as follows: (A) Score 0 for <10\% positive staining; (B) Score 1 for $10 \%$ to <40\% positive staining; (C) Score 2 for $40 \%$ to $<70 \%$ positive staining; (D) Score 3 for at least $70 \%$ positive staining. Original magnification $200 \times$. 

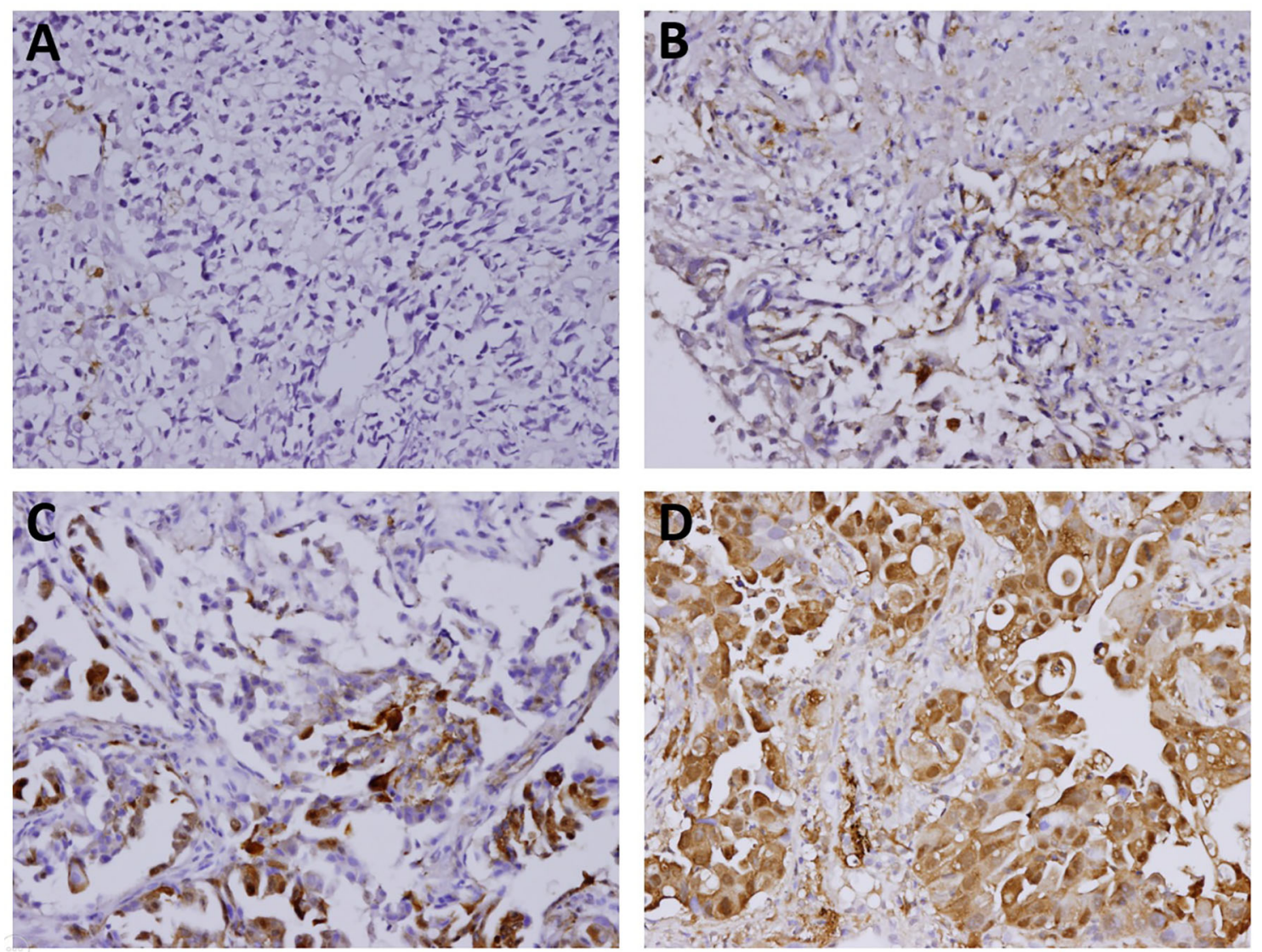

FIGURE 2 | The immunostaining features of tumor galectin-3 expression. The percentages of positive staining for galectin-3 in the tumor part were scored as follows: (A) Score 0 for <10\% positive staining; (B) Score 1 for 10\% to <40\% positive staining; (C) Score 2 for $40 \%$ to <70\% positive staining; (D) Score 3 for at least $70 \%$ positive staining. Original magnification 200x.

actuarial 1-year OS, LRPFS, and DMFS rates were 45.4\%, 38.2\%, and $23.2 \%$, respectively. The actuarial 2 -year OS, LRPFS, and DMFS rates were $17.7 \%, 7.6 \%$, and $7.7 \%$, respectively. The survival curves of OS, LRPFS, and DMFS for the whole population using the Kaplan-Meier method are presented as Figure 3.

The results of univariable and multivariable Cox regression analyses of possible prognostic factors for OS, LRPFS, and DMFS rates are listed in Tables $\mathbf{2 - 4}$, respectively. Overexpression of tumor galectin-1 was the most significant prognosticator to predict worse LRPFS in both univariable (hazard ratio $[\mathrm{HR}]=$ 3.18; $p=0.007)$ and multivariable analyses $(\mathrm{HR}=3.49 ; p=$ 0.022). The survival curve of LRPFS using the Kaplan-Meier method for overexpression versus non-overexpression of tumor galectin-1 is presented as Figure 4.

In addition, patients with overexpression of tumor galectin-1 had the trend of worse OS $(\mathrm{HR}=2.80 ; p=0.066)$ in multivariable analysis, and significantly worse DMFS $(\mathrm{HR}=2.43 ; p=0.035)$ in univariable analysis. The survival curve of DMFS using the Kaplan-Meier method for overexpression versus nonoverexpression of tumor galectin-1 is presented as Figure 5.
Other significant prognostic factors comprised male patients for worse OS $(p=0.048)$ and LRPFS $(p=0.047)$ in univariable analyses and overexpression of stroma galectin-3 for worse DMFS in univariable analysis $(p=0.040)$. Overexpression of tumor galectin-3 did not have any significant effect on survival outcomes in this cohort.

\section{DISCUSSION}

In this study, we found that overexpression of tumor galectin-1 was the most significant prognosticator to predict worse LRPFS in patients with lung adenocarcinoma after thoracic radiation therapy without concurrent chemotherapy. Since radiation therapy was the main locoregional therapy modality in this population, the result implied that tumor galectin-1 might be a biomarker to predict radiation response in the patients with lung adenocarcinoma.

Several studies had proposed the role of tumor galectin-1 in the clinical outcomes of non-small cell lung cancer. Carlini et al. (18) reported that high galectin-1 expression had a poorer OS in a cohort 
TABLE 1 | Patient characteristics $(N=41)$.

\begin{tabular}{|c|c|c|c|}
\hline Parameters & $\begin{array}{c}\text { All } \\
N=41, N(\%)\end{array}$ & $\begin{array}{c}\text { Tumor Galectin-1 } \\
\text { Score } 2-3, N=11, N(\%)\end{array}$ & $\begin{array}{c}\text { Tumor Galectin-3 } \\
\text { Score } 2-3, N=8, N(\%)\end{array}$ \\
\hline \multicolumn{4}{|l|}{ Gender } \\
\hline Female & $18(43.9)$ & $5(45.5)$ & $2(25.0)$ \\
\hline \multicolumn{4}{|l|}{ Age (years) } \\
\hline Median & 62.9 & 56.9 & 66.1 \\
\hline Range & $39.9-81.2$ & $45.9-70.2$ & $45.9-78.8$ \\
\hline \multicolumn{4}{|l|}{ T3-T4 } \\
\hline No & $9(22.0)$ & $4(36.4)$ & $2(25.0)$ \\
\hline Yes & 32 (78.0) & $7(63.6)$ & $6(75.0)$ \\
\hline \multicolumn{4}{|l|}{ N2-N3 } \\
\hline No & $13(31.7)$ & $2(18.2)$ & $2(25.0)$ \\
\hline Yes & $28(68.3)$ & $9(81.8)$ & $6(75.0)$ \\
\hline \multicolumn{4}{|c|}{ Tumor Galectin-1 } \\
\hline Score 2-3 & $35(85.4)$ & $10(90.9)$ & $7(87.5)$ \\
\hline \multicolumn{4}{|c|}{ Tumor Galectin-3 } \\
\hline Score 0-1 & $33(80.5)$ & $6(54.5)$ & - \\
\hline Score 2-3 & 8 (19.5) & $5(45.5)$ & - \\
\hline \multicolumn{4}{|c|}{ Stroma Galectin-3* } \\
\hline Score 0 & $25(61.0)$ & $2(18.2)$ & $2(25.0)$ \\
\hline Score 1 & $16(39.0)$ & $9(81.8)$ & $6(75.0)$ \\
\hline \multicolumn{4}{|l|}{ RT dose (Gy) } \\
\hline$<60$ & $12(29.3)$ & $1(9.1)$ & $2(25.0)$ \\
\hline$\geq 60$ & $29(70.7)$ & $10(90.9)$ & $8(75.0)$ \\
\hline \multicolumn{4}{|c|}{ Neoadjuvant Chemotherapy } \\
\hline No & $34(82.9)$ & $8(72.7)$ & $6(75.0)$ \\
\hline Yes & $7(17.1)$ & $3(27.3)$ & $2(25.0)$ \\
\hline
\end{tabular}

$R T$, radiation therapy

${ }^{*}$ No score 2-3 of galectin-3 expression in stroma.

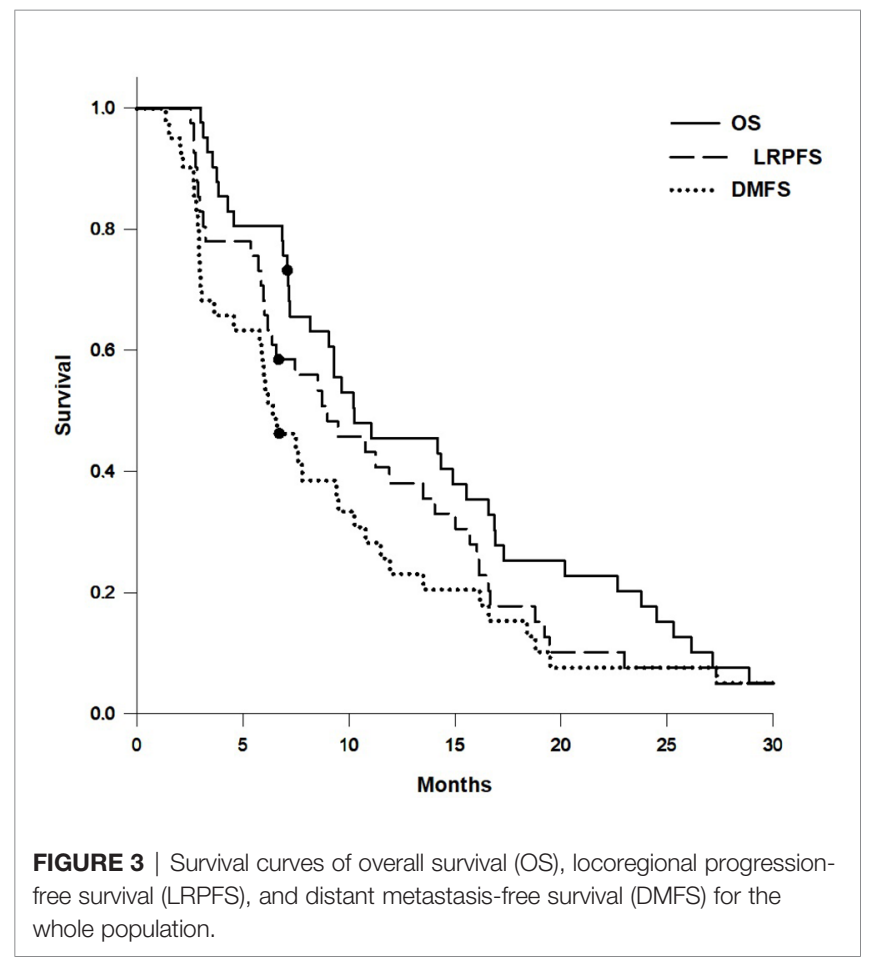

of 103 non-small cell lung cancer patients. Schulkens et al. (19) identified that galectin-1 was a significant prognostic factor for OS and progression-free survival (PFS) by performing extensive galectin expression profiling in a cohort of 87 non-small cell lung cancer patients. Zheng et al. (20) presented that positive expression of tumor galectin-1 could predict worse OS and PFS in a cohort of 96 surgically resected lung adenocarcinoma patients. In our study, we also found that overexpression of tumor galectin-1 had the trend of worse OS, and significantly worse LRPFS and DMFS in a cohort of 41 lung adenocarcinoma patients after radiation therapy. This study firstly reported the association of tumor galetin-1 in the clinical outcomes of lung adenocarcinoma after radiation therapy.

Several investigations had explored the possible mechanism of galectin-1 promoting tumor progression and chemoresistance in lung adenocarcinoma. Wu et al. (21) proposed that galectin-1 was involved in tumor invasion and metastasis by increasing matrix metalloproteinase (MMP)-9 and MMP-2 expression and reorganizing actin cytoskeletons. Chung et al. (9) discovered that galectin-1 promoted lung adenocarcinoma progression and chemoresistance by upregulating p38 MAPK, ERK, and cyclooxygenase-2 (COX-2). Zhou et al. (10) reported that galectin-1 overexpressed in CD133-positive lung adenocarcinoma cells, featuring higher invasive and metastatic abilities, and played a major role in tumorigenesis and invasiveness via COX-2/PGE2 and 
TABLE 2 | Univariable and multivariable Cox regression analyses of OS.

\begin{tabular}{|c|c|c|c|c|c|c|}
\hline \multirow[t]{2}{*}{ Variables } & \multicolumn{3}{|c|}{ Univariable analysis } & \multicolumn{3}{|c|}{ Multivariable analysis } \\
\hline & HR & $95 \% \mathrm{Cl}$ & $p$ & HR & $95 \% \mathrm{Cl}$ & $p$ \\
\hline Male & 1.95 & $1.01-3.76$ & 0.048 & 1.89 & $0.76-4.69$ & 0.171 \\
\hline Age $\geq 65$ years & 1.69 & $0.90-3.19$ & 0.105 & 1.47 & $0.72-3.01$ & 0.294 \\
\hline T3-T4 & 0.86 & $0.40-1.83$ & 0.690 & 0.73 & $0.29-1.82$ & 0.496 \\
\hline N2-N3 & 1.37 & $0.67-2.82$ & 0.388 & 0.97 & $0.37-2.53$ & 0.953 \\
\hline Tumor Galectin-1 & 1.75 & $0.83-3.68$ & 0.142 & 2.80 & $0.93-8.40$ & 0.066 \\
\hline Stroma Galectin-1 & 2.02 & $0.78-5.26$ & 0.148 & 1.51 & $0.44-5.19$ & 0.516 \\
\hline Tumor Galectin-3 & 1.24 & $0.56-2.74$ & 0.595 & 0.59 & $0.20-1.69$ & 0.326 \\
\hline Stroma Galectin-3 & 1.26 & $0.66-2.41$ & 0.491 & 0.95 & $0.36-2.47$ & 0.912 \\
\hline RT dose $\geq 60$ Gy & 0.69 & $0.34-1.41$ & 0.312 & 0.56 & $0.24-1.32$ & 0.184 \\
\hline Neoadjuvant Chemotherapy & 0.65 & $0.28-1.49$ & 0.307 & 0.65 & $0.24-1.73$ & 0.386 \\
\hline
\end{tabular}

OS, overall survival; RT, radiation therapy; HR, hazard ratio; Cl, confidence interval. The $p$-values were calculated using log-rank test.

TABLE 3 | Univariable and multivariable Cox regression analyses of LRPFS.

\begin{tabular}{|c|c|c|c|c|c|c|}
\hline \multirow[t]{2}{*}{ Variables } & \multicolumn{3}{|c|}{ Univariable analysis } & \multicolumn{3}{|c|}{ Multivariable analysis } \\
\hline & HR & $95 \% \mathrm{Cl}$ & $p$ & HR & $95 \% \mathrm{Cl}$ & $p$ \\
\hline Male & 1.95 & $1.01-3.77$ & 0.047 & 2.26 & $0.85-6.01$ & 0.101 \\
\hline Age $\geq 65$ years & 1.80 & $0.94-3.43$ & 0.076 & 1.43 & $0.69-2.97$ & 0.344 \\
\hline T3-T4 & 0.81 & $0.38-1.72$ & 0.581 & 0.75 & $0.30-1.92$ & 0.553 \\
\hline N2-N3 & 1.59 & $0.78-3.25$ & 0.205 & 1.09 & $0.42-2.81$ & 0.860 \\
\hline Tumor Galectin-1 & 3.18 & $1.37-7.41$ & 0.007 & 3.49 & $1.20-10.14$ & 0.022 \\
\hline Stroma Galectin-1 & 2.20 & $0.85-5.70$ & 0.106 & 1.47 & $0.44-4.96$ & 0.531 \\
\hline Tumor Galectin-3 & 1.71 & $0.78-3.76$ & 0.184 & 0.69 & $0.25-1.90$ & 0.472 \\
\hline Stroma Galectin-3 & 1.48 & $0.77-2.85$ & 0.244 & 1.27 & $0.50-3.22$ & 0.618 \\
\hline RT dose $\geq 60$ Gy & 0.75 & $0.37-1.51$ & 0.423 & 0.63 & $0.27-1.47$ & 0.281 \\
\hline $\begin{array}{l}\text { Neoadjuvant } \\
\text { Chemotherapy }\end{array}$ & 0.95 & $0.42-2.17$ & 0.906 & 1.13 & $0.41-3.07$ & 0.814 \\
\hline
\end{tabular}

LRPFS, locoregional progression-free survival; RT, radiation therapy; HR, hazard ratio; Cl, confidence interval. The p-values were calculated using log-rank test.

TABLE 4 | Univariable and multivariable Cox regression analyses of DMFS.

\begin{tabular}{|c|c|c|c|c|c|c|}
\hline Variables & \multicolumn{3}{|c|}{ Univariable analysis } & \multicolumn{3}{|c|}{ Multivariable analysis } \\
\hline Male & 1.25 & $0.65-2.41$ & 0.501 & 1.69 & $0.70-4.07$ & 0.242 \\
\hline T3-T4 & 0.88 & $0.41-1.87$ & 0.739 & 1.17 & $0.46-2.97$ & 0.738 \\
\hline N2-N3 & 1.54 & $0.76-3.12$ & 0.234 & 1.48 & $0.56-3.91$ & 0.434 \\
\hline Tumor Galectin-1 & 2.43 & $1.06-5.54$ & 0.035 & 1.76 & $0.62-4.98$ & 0.290 \\
\hline Stroma Galectin-3 & 2.02 & $1.03-3.96$ & 0.040 & 2.22 & $0.90-5.47$ & 0.084 \\
\hline RT dose $\geq 60$ Gy & 1.13 & $0.56-2.27$ & 0.730 & 1.19 & $0.51-2.76$ & 0.687 \\
\hline Neoadjuvant Chemotherapy & 1.52 & $0.66-3.48$ & 0.327 & 2.47 & $0.87-7.04$ & 0.090 \\
\hline
\end{tabular}

DMFS, distant metastasis-free survival; RT, radiation therapy; HR, hazard ratio; Cl, confidence interval. The $p$-values were calculated using log-rank test.

AKT/mTOR pathways. Zhang et al. (22) found that galectin-1 gene silencing would improve the sensitivity of lung adenocarcinoma cells to cisplatin in vivo and in vitro. However, there was a lack of studies to explore the possible mechanism of radioresistance in lung adenocarcinoma, which prompted us to go into study.

As for galectin-3, previous studies had showed the inconsistent association of galectin-3 in the clinical outcomes of non-small cell lung cancer. Mathieu et al. (23) reported that nuclear galectin-3 expression is a predictive factor of recurrence in a cohort of 286 surgically resected lung adenocarcinoma and squamous cell carcinoma patients. Kosacka et al. (24) revealed no prognostic value of galectin-3 expression in non-small cell lung cancer. Kataoka et al. (25) presented that galectin-3 expression in tumor cells could serve as a predictive factor for recurrence (mainly distal recurrence) in patients with nonsmall cell lung cancer. Kao et al. reported that low galectin-3 expression is associated with metastasis in surgically resected T1 lung adenocarcinoma. In our study, we found that 


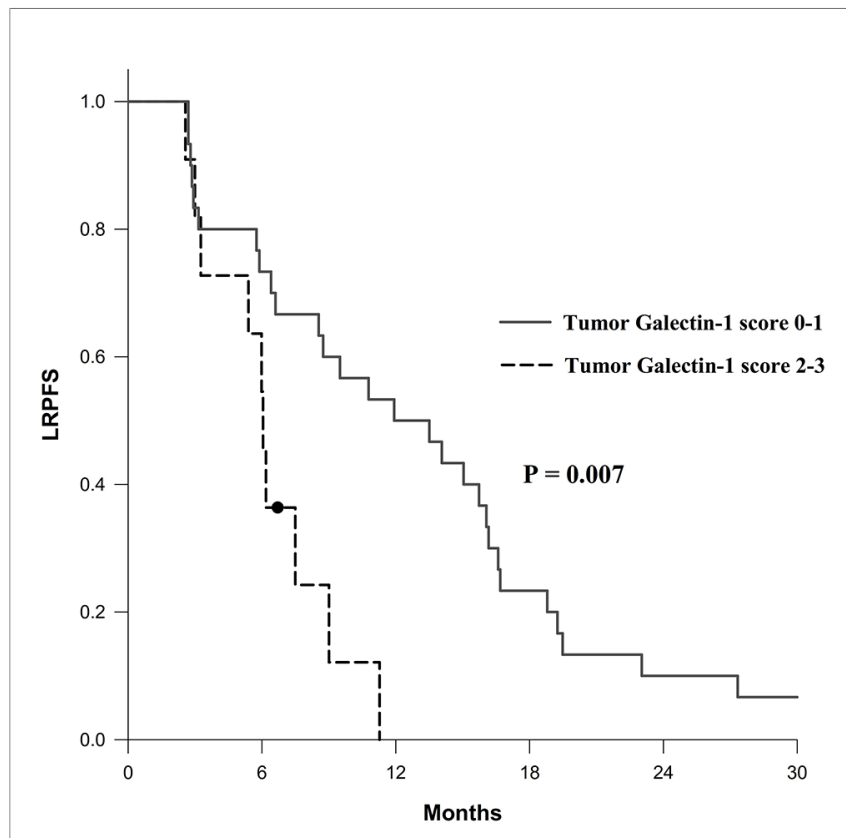

FIGURE 4 | Survival curve of locoregional progression-free survival (LRPFS) according to overexpression (score 2-3) versus non-overexpression (score 0-1) of tumor galectin-1. The $p$-value was calculated using log-rank test.

overexpression of stroma galectin-3 would predict worse DMFS in univariable analysis; however, overexpression of tumor galectin-3 had no significant effect on the survival outcomes in the patients with lung adenocarcinoma after radiation therapy.

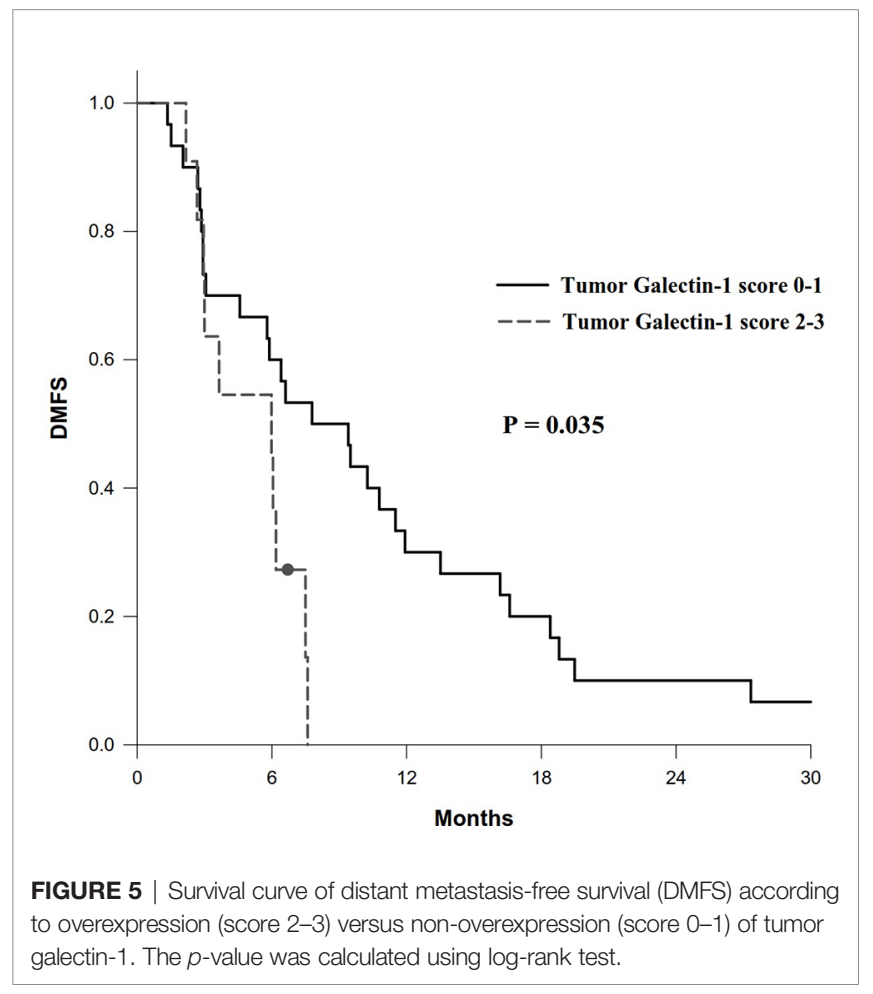

The tumor stroma could change during malignancy and might have an influence on tumor progression (26). We were curious about the percentage of overexpression of galectin-1 and galectin-3 and their prognostic impact on survival outcomes. In this study, we found higher overexpression of galectin-1 (85.4\%) than galectin-3 (39.0\%) in the stroma part; however, there was no prognostic impact of stroma galectin-1. Overexpression of stroma galectin-3 could predict worse DMFS in univariable analysis but not in multivariable analysis.

The patient being male was the other significant prognostic factor for worse OS and LRPFS in univariable analyses in our study. Previous literature also showed the same finding. Sakurai et al. (27) reported that male patients had significant worse OS and disease-specific survival in a large cohort of 8,168 lung adenocarcinoma patients. Watanabe et al. (28) revealed that male patients had obviously shorter disease-free interval in lung adenocarcinoma. Kawaguchi et al. (29) presented that male patients had worse OS in the never-smokers with nonsmall cell lung cancer mainly comprising adenocarcinoma.

The survival outcomes in our study from patients undergoing conventional 2D radiotherapy (2DRT) in the old non-concurrent chemotherapy era were much poorer than those in the report from patients in the concurrent chemotherapy era. The 2-year OS rate in our study was only $17.7 \%$, which was only one-half of $35.6 \%$ reported from a meta-analysis (30) including patients with concurrent chemotherapy. With application of much more conformal and precise radiation therapy techniques in the modern era, the reported survival outcomes in the patients with locally advanced non-small cell lung cancer were much better than those reported in the conventional 2DRT era. In a well-known clinical trial (31) of concurrent chemoradiotherapy with or without adjuvant durvalumab immunotherapy, the 2 year OS rate could be as high as $55.6 \%$ in the placebo arm and $66.3 \%$ in the durvalumab arm.

The main limitations of this study are its limited sample size and the study being retrospective in nature, which may include inherent selection bias. In addition, the enrolled patients were in the old era, and some parts of the charts might be destroyed. The patient characteristics including clinical (such as performance status) or pathological variables (such as tumor grade) are not available, which may bias our results. Finally, the result of treatment outcome is limited to patients without concurrent chemotherapy, which cannot be presented as the treatment outcome of current standard therapy (concurrent chemoradiotherapy with adjuvant durvalumab immunotherapy).

\section{CONCLUSION}

We report the treatment outcome of patients with lung adenocarcinoma after thoracic radiation therapy without concurrent chemotherapy. The overexpression of tumor galectin-1 is the most significant prognosticator for poor LRPFS. Future research on the mechanism of galectin-1 affecting radiation response in lung adenocarcinoma may be worth exploring. 


\section{DATA AVAILABILITY STATEMENT}

The datasets generated for this study are available on request to the corresponding authors.

\section{ETHICS STATEMENT}

The studies involving human participants were reviewed and approved by Chang Gung Medical Foundation Institutional Review Board. The ethics committee waived the requirement of written informed consent for participation.

\section{AUTHOR CONTRIBUTIONS}

$\mathrm{C}-\mathrm{CHu}, \mathrm{I}-\mathrm{CC}, \mathrm{C}-\mathrm{CHs}$, and $\mathrm{E}-\mathrm{YH}$ were involved in the conception and design. C-CHu, I-CC, Y-LS, H-LL, Y-CC, and

\section{REFERENCES}

1. Health Promotion Administration and Ministry of Health and Welfare. Taiwan Cancer Registry Annual Report 2018. Available at: https:/www.hpa.gov.tw/ Pages/Detail.aspx?nodeid=269\&pid=13498 (Accessed January 17, 2022).

2. Vinod SK, Hau E. Radiotherapy Treatment for Lung Cancer: Current Status and Future Directions. Respirology (2020) 25(Suppl 2):61-71. doi: 10.1111/ resp. 13870

3. Brown S, Banfill K, Aznar MC, Whitehurst P, Faivre Finn C. The Evolving Role of Radiotherapy in Non-Small Cell Lung Cancer. Br J Radiol (2019) 92 (1104):20190524. doi: 10.1259/bjr.20190524

4. Shah JL, Loo BWJr. Stereotactic Ablative Radiotherapy for Early-Stage Lung Cancer. Semin Radiat Oncol (2017) 27(3):218-28. doi: 10.1016/j.semradonc. 2017.03.001

5. Christodoulou M, Bayman N, McCloskey P, Rowbottom C, Faivre-Finn C. New Radiotherapy Approaches in Locally Advanced Non-Small Cell Lung Cancer. Eur J Cancer (2014) 50(3):525-34. doi: 10.1016/j.ejca.2013. 11.027

6. Zhou Y, Yu F, Zhao Y, Zeng Y, Yang X, Chu L, et al. A Narrative Review of Evolving Roles of Radiotherapy in Advanced Non-Small Cell Lung Cancer: From Palliative Care to Active Player. Transl Lung Cancer Res (2020) 9 (6):2479-93. doi: 10.21037/tlcr-20-1145

7. Mantovani C, Gastino A, Cerrato M, Badellino S, Ricardi U, Levis M. Modern Radiation Therapy for the Management of Brain Metastases From Non-Small Cell Lung Cancer: Current Approaches and Future Directions. Front Oncol (2021) 11:772789. doi: 10.3389/fonc.2021.772789

8. Hsu YL, Wu CY, Hung JY, Lin YS, Huang MS, Kuo PL. Galectin-1 Promotes Lung Cancer Tumor Metastasis by Potentiating Integrin Alpha6beta4 and Notch1/Jagged2 Signaling Pathway. Carcinogenesis (2013) 34(6):1370-81. doi: 10.1093/carcin/bgt040

9. Chung LY, Tang SJ, Sun GH, Chou TY, Yeh TS, Yu SL, et al. Galectin-1 Promotes Lung Cancer Progression and Chemoresistance by Upregulating P38 MAPK, ERK, and Cyclooxygenase-2. Clin Cancer Res (2012) 18 (15):4037-47. doi: 10.1158/1078-0432.CCR-11-3348

10. Zhou X, Li D, Wang X, Zhang B, Zhu H, Zhao J. Galectin-1 Is Overexpressed in CD133+ Human Lung Adenocarcinoma Cells and Promotes Their Growth and Invasiveness. Oncotarget (2015) 6(5):3111-22. doi: 10.18632/ oncotarget.3076

11. Chung LY, Tang SJ, Wu YC, Sun GH, Liu HY, Sun KH. Galectin-3 Augments Tumor Initiating Property and Tumorigenicity of Lung Cancer Through Interaction With Beta-Catenin. Oncotarget (2015) 6(7):4936-52. doi: 10.18632/oncotarget.3210
J-YC were involved in the preparation, analysis, and interpretation of the data. $\mathrm{C}-\mathrm{CHu}$ and I-CC drafted the paper. $\mathrm{C}-\mathrm{CHs}$ and E-YH revised it critically for intellectual content. All authors contributed to the article and approved the submitted version.

\section{FUNDING}

The work was supported in part by grants from Chang Gung Medical Foundation/Chang Gung Memorial Hospital (CMRPG8H1001 and CMRPG8H1002) to C-CHu.

\section{ACKNOWLEDGMENTS}

We thank Chang Gung Medical Foundation Kaohsiung Chang Gung Memorial Hospital Tissue Bank Core Lab (CLRPG8I0031) for excellent technical support.
12. Kuo HY, Hsu HT, Chen YC, Chang YW, Liu FT, Wu CW. Galectin-3 Modulates the EGFR Signalling-Mediated Regulation of Sox2 Expression via C-Myc in Lung Cancer. Glycobiology (2016) 26(2):155-65. doi: 10.1093/ glycob/cwv088

13. Zhou W, Chen X, Hu Q, Chen X, Chen Y, Huang L. Galectin-3 Activates TLR4/NF-kappaB Signaling to Promote Lung Adenocarcinoma Cell Proliferation Through Activating lncRNA-NEAT1 Expression. BMC Cancer (2018) 18(1):580. doi: 10.1186/s12885-018-4461-z

14. Huang EY, Chen YF, Chen YM, Lin IH, Wang CC, Su WH, et al. A Novel Radioresistant Mechanism of Galectin-1 Mediated by H-Ras-Dependent Pathways in Cervical Cancer Cells. Cell Death Dis 3:e251. doi: 10.1038/ cddis. 2011.120

15. Huang EY, Chanchien CC, Lin H, Wang CC, Wang CJ, Huang CC. Galectin-1 Is an Independent Prognostic Factor for Local Recurrence and Survival After Definitive Radiation Therapy for Patients With Squamous Cell Carcinoma of the Uterine Cervix. Int J Radiat Oncol Biol Phys (2013) 87(5):975-82. doi: 10.1016/j.ijrobp.2013.08.037

16. Chou SY, Yen SL, Huang CC, Huang EY. Galectin-1 Is a Poor Prognostic Factor in Patients With Glioblastoma Multiforme After Radiotherapy. BMC Cancer (2018) 18(1):105. doi: 10.1186/s12885-018-4025-2

17. Li SH, Chen YH, Lu HI, Lo CM, Huang CC, Wang YM, et al. Galectin-1 Expression Is Associated With the Response and Survival Following Preoperative Chemoradiotherapy in Locally Advanced Esophageal Squamous Cell Carcinoma. Cancers (2021) 13(13):3147. doi: 10.3390/cancers13133147

18. Carlini MJ, Roitman P, Nunez M, Pallotta MG, Boggio G, Smith D, et al. Clinical Relevance of Galectin-1 Expression in Non-Small Cell Lung Cancer Patients. Lung Cancer (2014) 84(1):73-8. doi: 10.1016/j.lungcan.2014.01.016

19. Schulkens IA, Heusschen R, van den Boogaart V, van Suylen RJ, Dingemans AM, Griffioen AW, et al. Galectin Expression Profiling Identifies Galectin-1 and Galectin-9Delta5 as Prognostic Factors in Stage I/II Non-Small Cell Lung Cancer. PLoS One (2014) 9(9):e107988. doi: 10.1371/journal.pone.0107988

20. Zheng H, Cui Y, Li X, Du B, Li Y. Prognostic Significance of (18)F-FDG PET/ CT Metabolic Parameters and Tumor Galectin-1 Expression in Patients With Surgically Resected Lung Adenocarcinoma. Clin Lung Cancer (2019) 20 (6):420-8. doi: 10.1016/j.cllc.2019.04.002

21. Wu MH, Hong TM, Cheng HW, Pan SH, Liang YR, Hong HC, et al. Galectin1-Mediated Tumor Invasion and Metastasis, Up-Regulated Matrix Metalloproteinase Expression, and Reorganized Actin Cytoskeletons. Mol Cancer Res (2009) 7(3):311-8. doi: 10.1158/1541-7786.MCR-08-0297

22. Zhang L, Liu X, Tang Z, Li X, Wang G. Reversal of Galectin-1 Gene Silencing on Resistance to Cisplatin in Human Lung Adenocarcinoma A549 Cells. BioMed Pharmacother (2016) 83:265-70. doi: 10.1016/j.biopha.2016.06.030 
23. Mathieu A, Saal I, Vuckovic A, Ransy V, Vereerstraten P, Kaltner H, et al. Nuclear Galectin-3 Expression Is an Independent Predictive Factor of Recurrence for Adenocarcinoma and Squamous Cell Carcinoma of the Lung. Mod Pathol (2005) 18(9):1264-71. doi: 10.1038/modpathol.3800416

24. Kosacka M, Piesiak P, Kowal A, Golecki M, Jankowska R. Galectin-3 and Cyclin D1 Expression in Non-Small Cell Lung Cancer. J Exp Clin Cancer Res (2011) 30:101. doi: 10.1186/1756-9966-30-101

25. Kataoka Y, Igarashi T, Ohshio Y, Fujita T, Hanaoka J. Predictive Importance of Galectin-3 for Recurrence of Non-Small Cell Lung Cancer. Gen Thorac Cardiovasc Surg (2019) 67(8):704-11. doi: 10.1007/s11748-019-01074-x

26. Bremnes RM, Donnem T, Al-Saad S, Al-Shibli K, Andersen S, Sirera R, et al. The Role of Tumor Stroma in Cancer Progression and Prognosis: Emphasis on Carcinoma-Associated Fibroblasts and Non-Small Cell Lung Cancer. J Thorac Oncol (2011) 6(1):209-17. doi: 10.1097/JTO. 0b013e3181f8albd

27. Sakurai H, Asamura H, Goya T, Eguchi K, Nakanishi Y, Sawabata N, et al. Survival Differences by Gender for Resected Non-Small Cell Lung Cancer: A Retrospective Analysis of 12,509 Cases in a Japanese Lung Cancer Registry Study. J Thorac Oncol (2010) 5(10):1594-601. doi: 10.1097/JTO. 0b013e3181f1923b

28. Watanabe K, Sakamaki K, Nishii T, Yamamoto T, Maehara T, Nakayama H, et al. Gender Differences in the Recurrence Timing of Patients Undergoing Resection for Non-Small Cell Lung Cancer. Asian Pac J Cancer Prev (2018) 19 (3):719-24. doi: 10.22034/APJCP.2018.19.3.719

29. Kawaguchi T, Takada M, Kubo A, Matsumura A, Fukai S, Tamura A, et al. Gender, Histology, and Time of Diagnosis Are Important Factors for Prognosis: Analysis of 1499 Never-Smokers With Advanced Non-Small Cell
Lung Cancer in Japan. J Thorac Oncol (2010) 5(7):1011-7. doi: 10.1097/ JTO.0b013e3181dc213e

30. Auperin A, Le Pechoux C, Rolland E, Curran WJ, Furuse K, Fournel P, et al. Meta-Analysis of Concomitant Versus Sequential Radiochemotherapy in Locally Advanced Non-Small-Cell Lung Cancer. J Clin Oncol (2010) 28 (13):2181-90. doi: 10.1200/JCO.2009.26.2543

31. Antonia SJ, Villegas A, Daniel D, Vicente D, Murakami S, Hui R, et al. Overall Survival With Durvalumab After Chemoradiotherapy in Stage III NSCLC. N Engl J Med (2018) 379(24):2342-50. doi: 10.1056/NEJMoa1809697

Conflict of Interest: The authors declare that the research was conducted in the absence of any commercial or financial relationships that could be construed as a potential conflict of interest.

Publisher's Note: All claims expressed in this article are solely those of the authors and do not necessarily represent those of their affiliated organizations, or those of the publisher, the editors and the reviewers. Any product that may be evaluated in this article, or claim that may be made by its manufacturer, is not guaranteed or endorsed by the publisher.

Copyright (c) 2022 Huang, Chuang, Su, Luo, Chang, Chen, Hsiao and Huang. This is an open-access article distributed under the terms of the Creative Commons Attribution License (CC BY). The use, distribution or reproduction in other forums is permitted, provided the original author(s) and the copyright owner(s) are credited and that the original publication in this journal is cited, in accordance with accepted academic practice. No use, distribution or reproduction is permitted which does not comply with these terms. 